\title{
Overview of the Space Launch System Ascent Aeroacoustic Environment Test Program
}

5 January, 2016

Andrew J. Herron, William A. Crosby, and Darren K. Reed NASA/George C. Marshall Space Flight Center

$54^{\text {th }}$ AIAA Aerospace Sciences Meeting

4-8 January, 2016

San Diego, California 


\section{Overview}

- Objectives

$\checkmark$ Test Models

- Instrumentation

- Test Details

- Data Analysis

- Results

- Conclusions 


\section{Objectives}

- Primary Objective

- Collect sufficient high frequency fluctuating pressure time histories for baseline SLS Block 1 and 1B models to determine ascent aeroacoustic external environments

- Secondary Objectives

- Collect surface static pressure measurements

- Compartment venting

- Protuberance airloads

- CFD substantiation

- MPCV flush air data system calibration

- Study alternate booster nose caps (NASA Engineering and Safety Center)

- Determine buffet and acoustic mitigation option (BMO and AMO) effectiveness 


\section{Test Models}

- $2.5 \%$ Full Stack

- Block 1 SLS-10003 OML with updates for -10004 OML

- Block 1B SLS-27000 (cargo) OML

- Five NESC nose caps, $21 \mathrm{BMO}$ and AMO

- $4 \%$ Forebody (truncated at SRB tip)

- Block 1 SLS-10003 OML with updates for -10004 OML

- Block 1B SLS-28000 (crew) OML

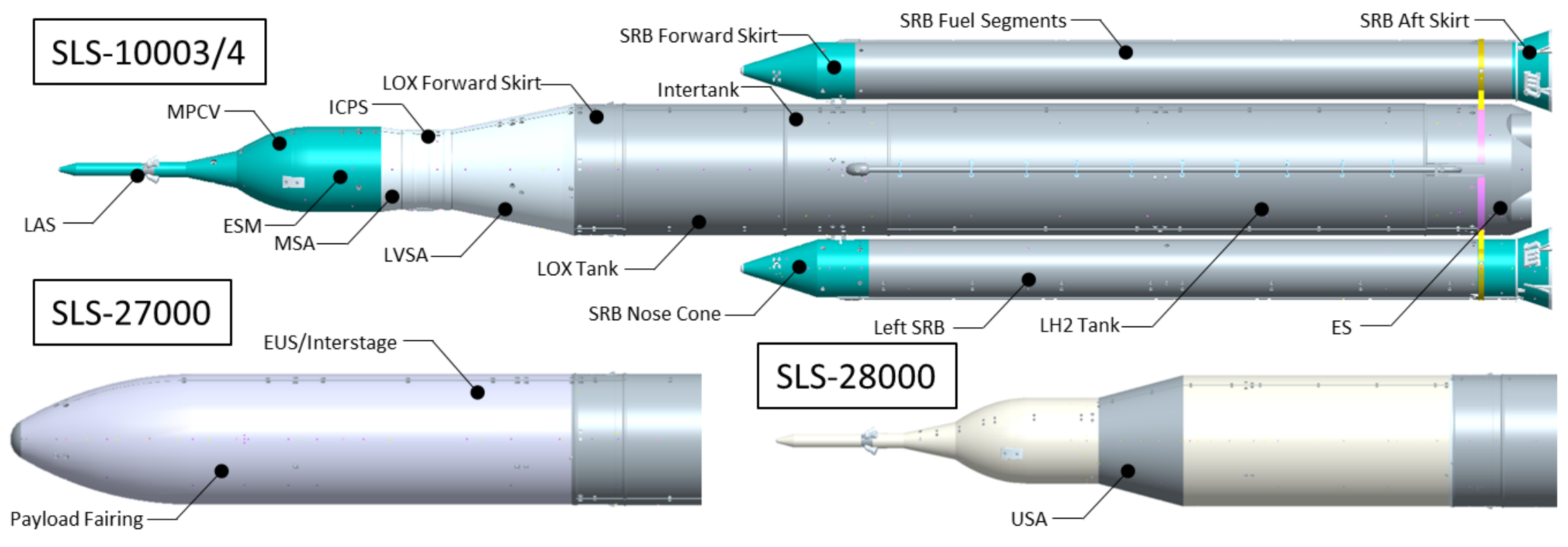




\section{5\% Models}

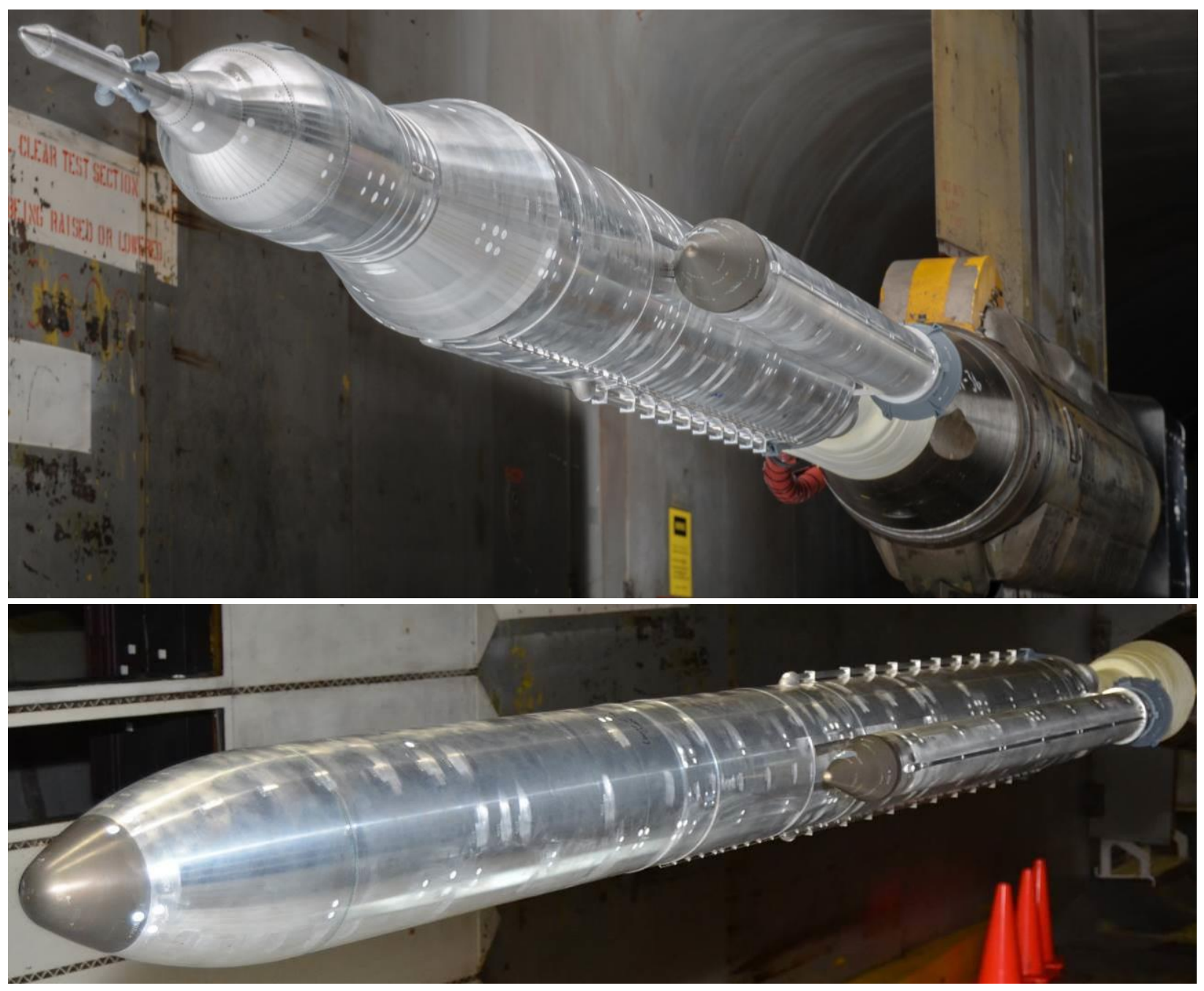




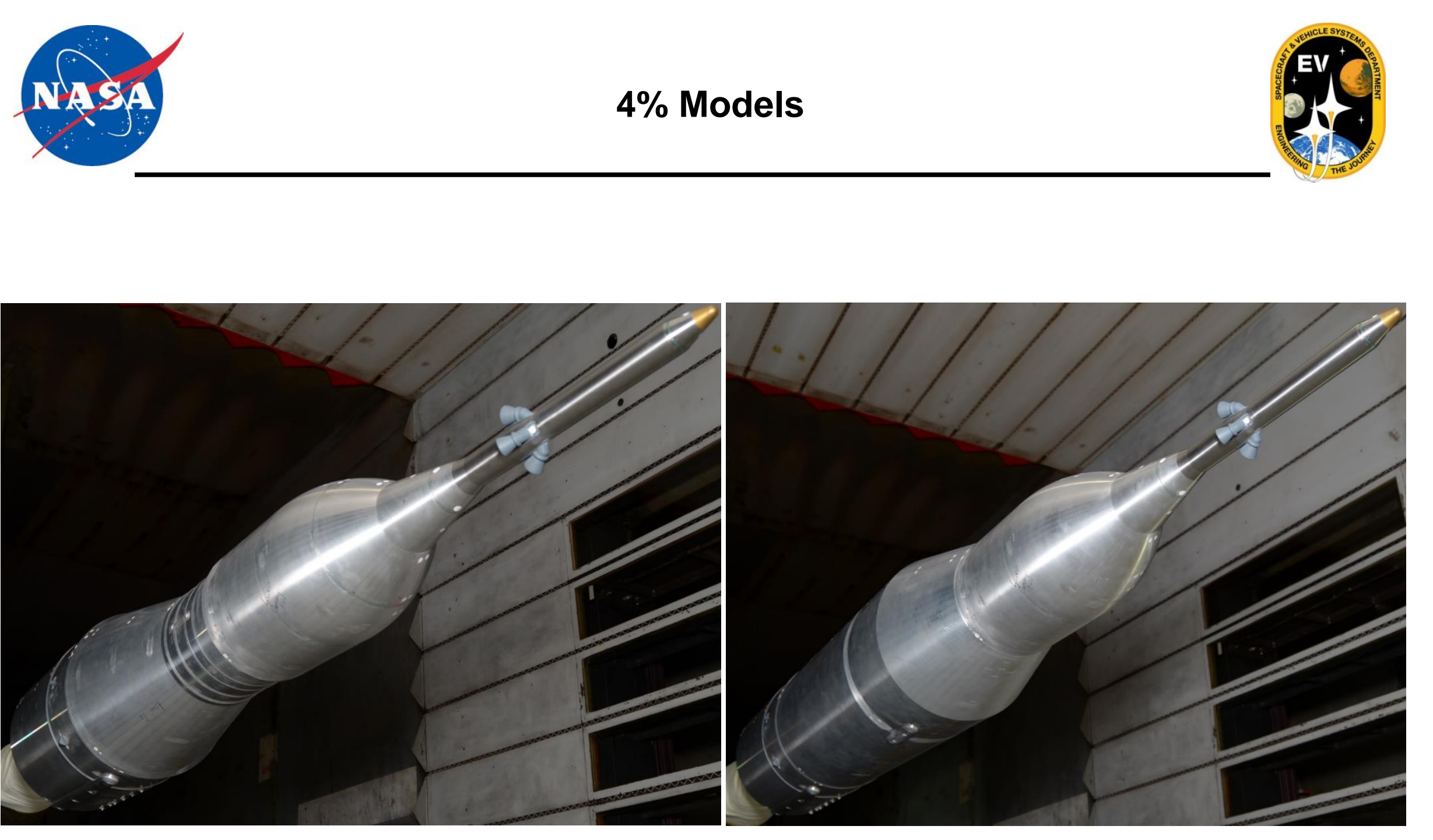




\section{NESC Booster Nose Caps}
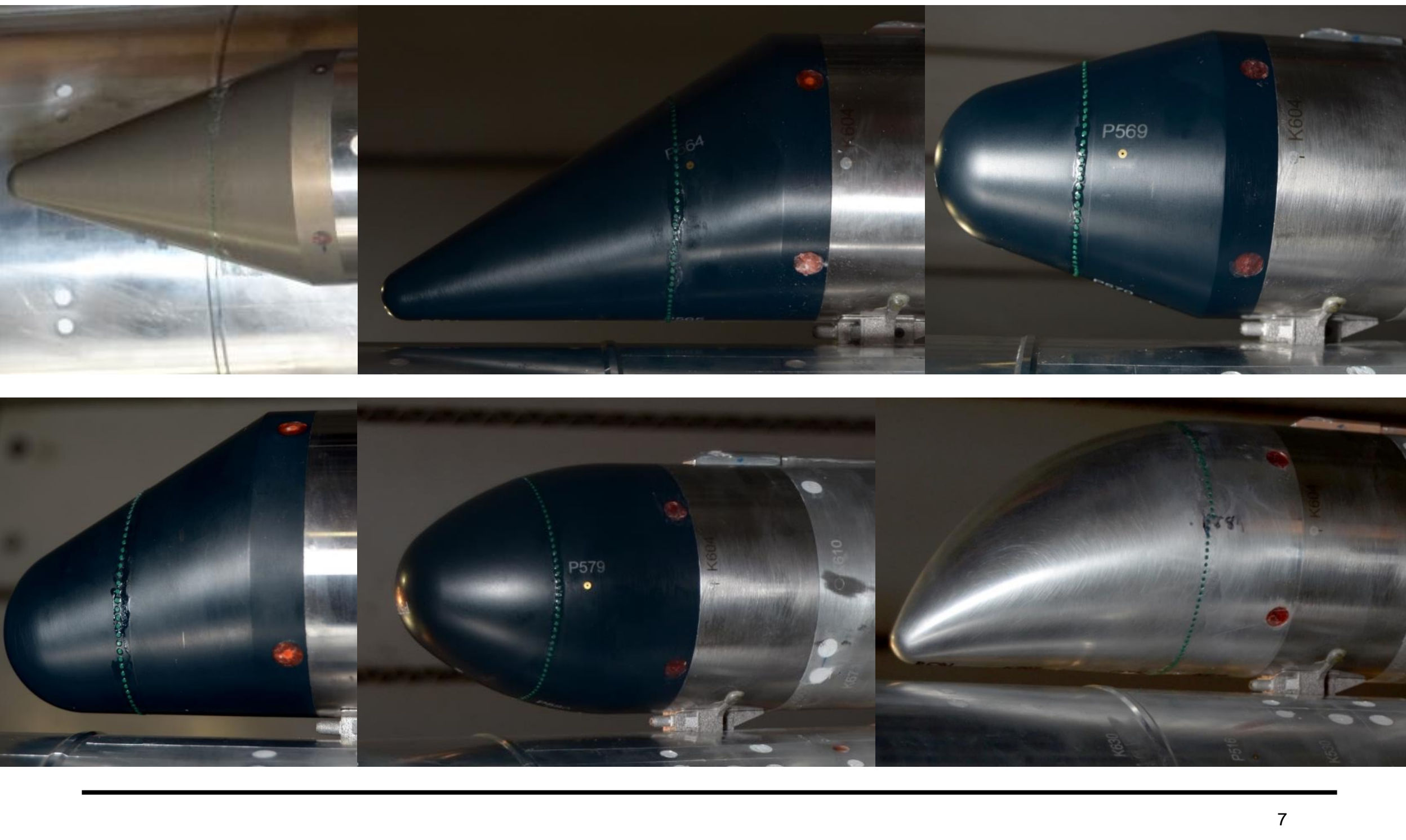


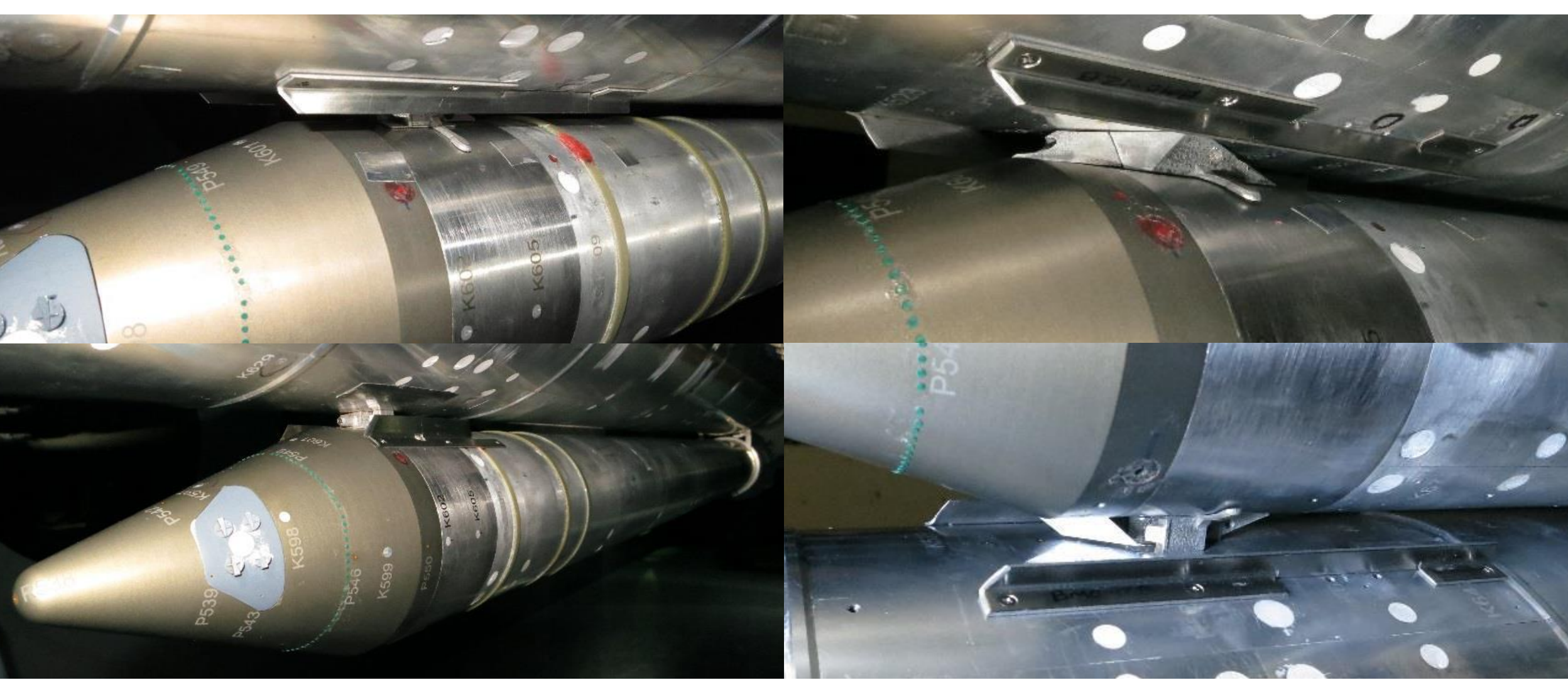




\section{Full Stack Protuberances}

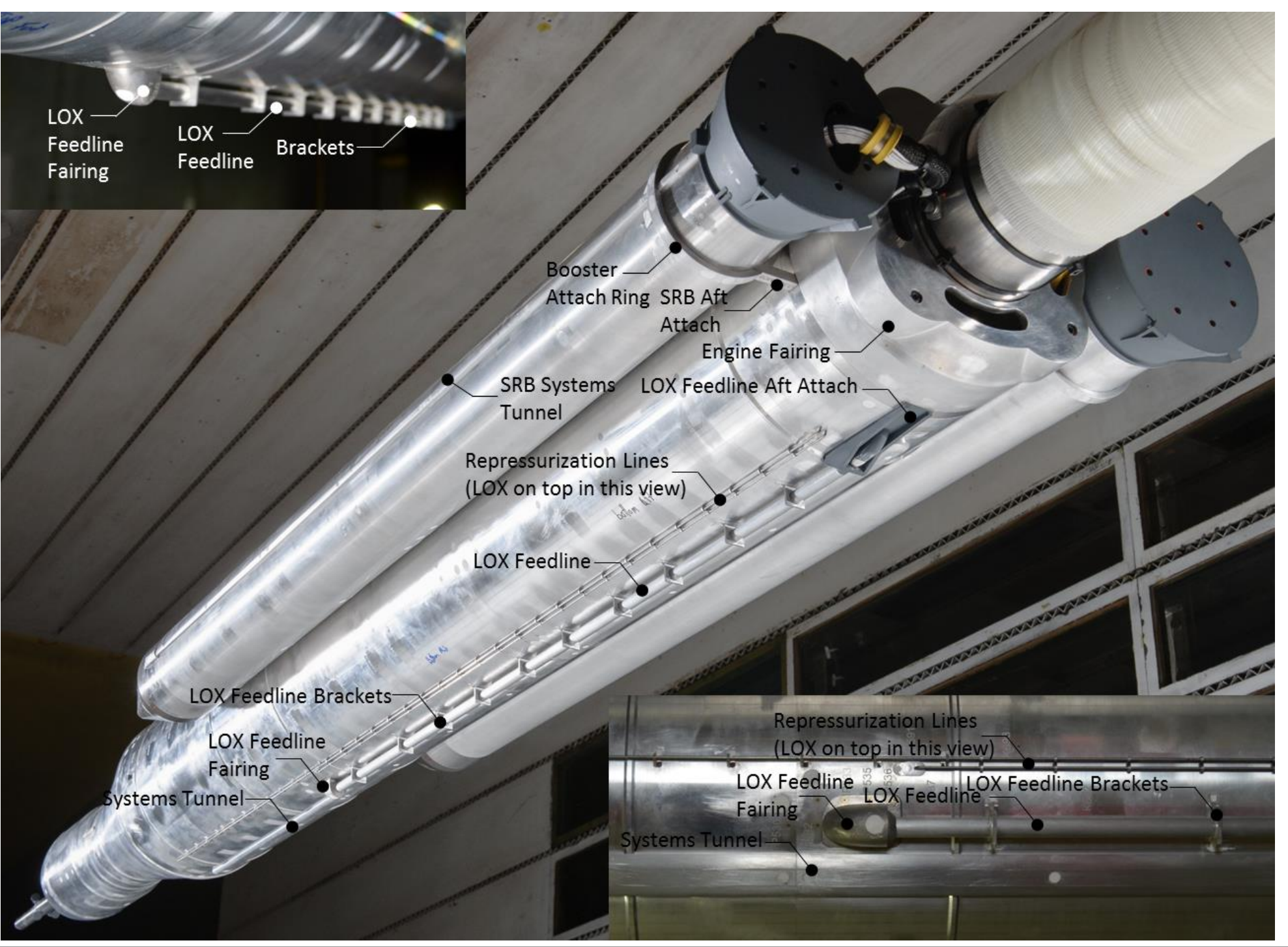




\section{Full Stack Protuberances}

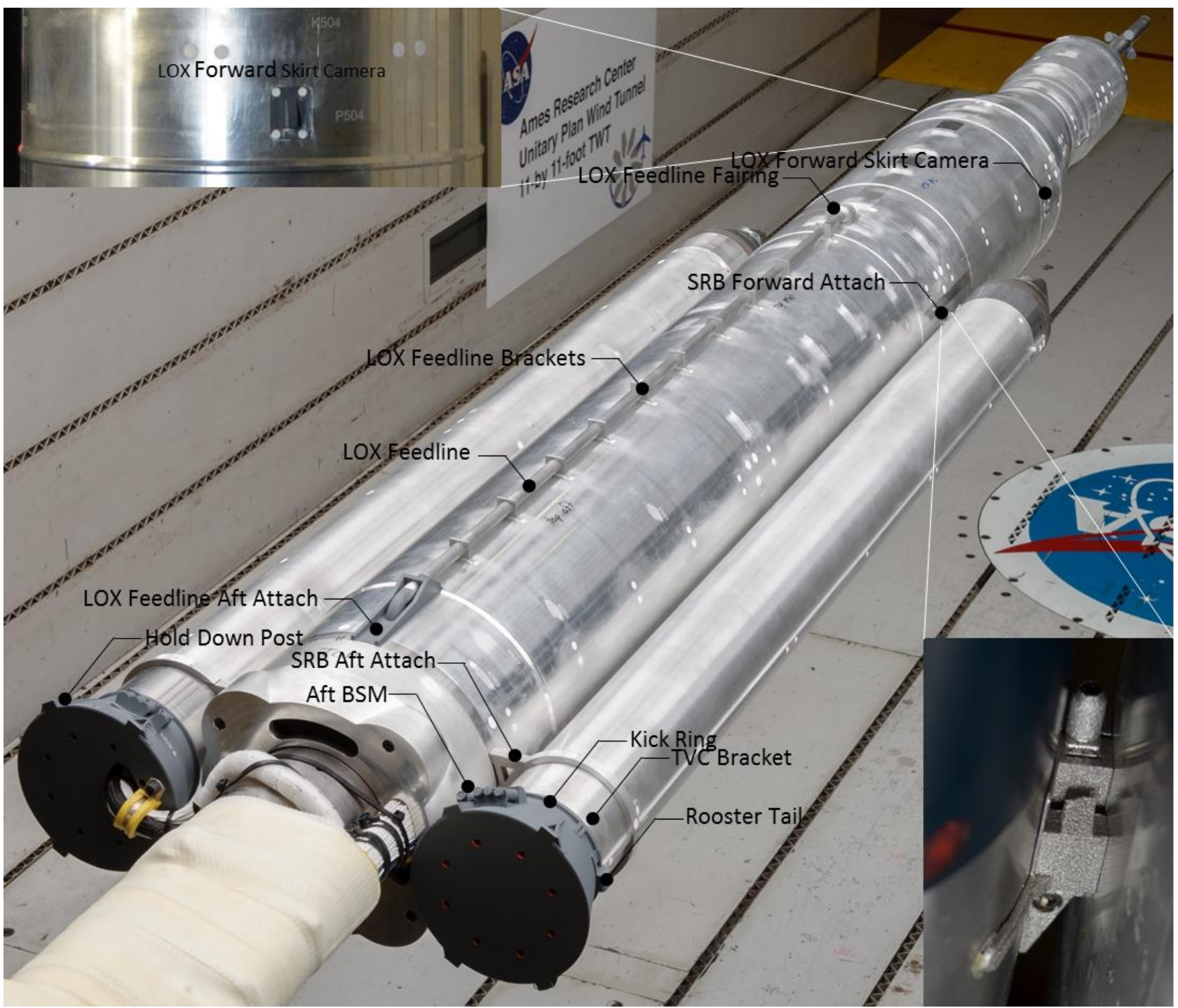




\section{Forebody Protuberances}
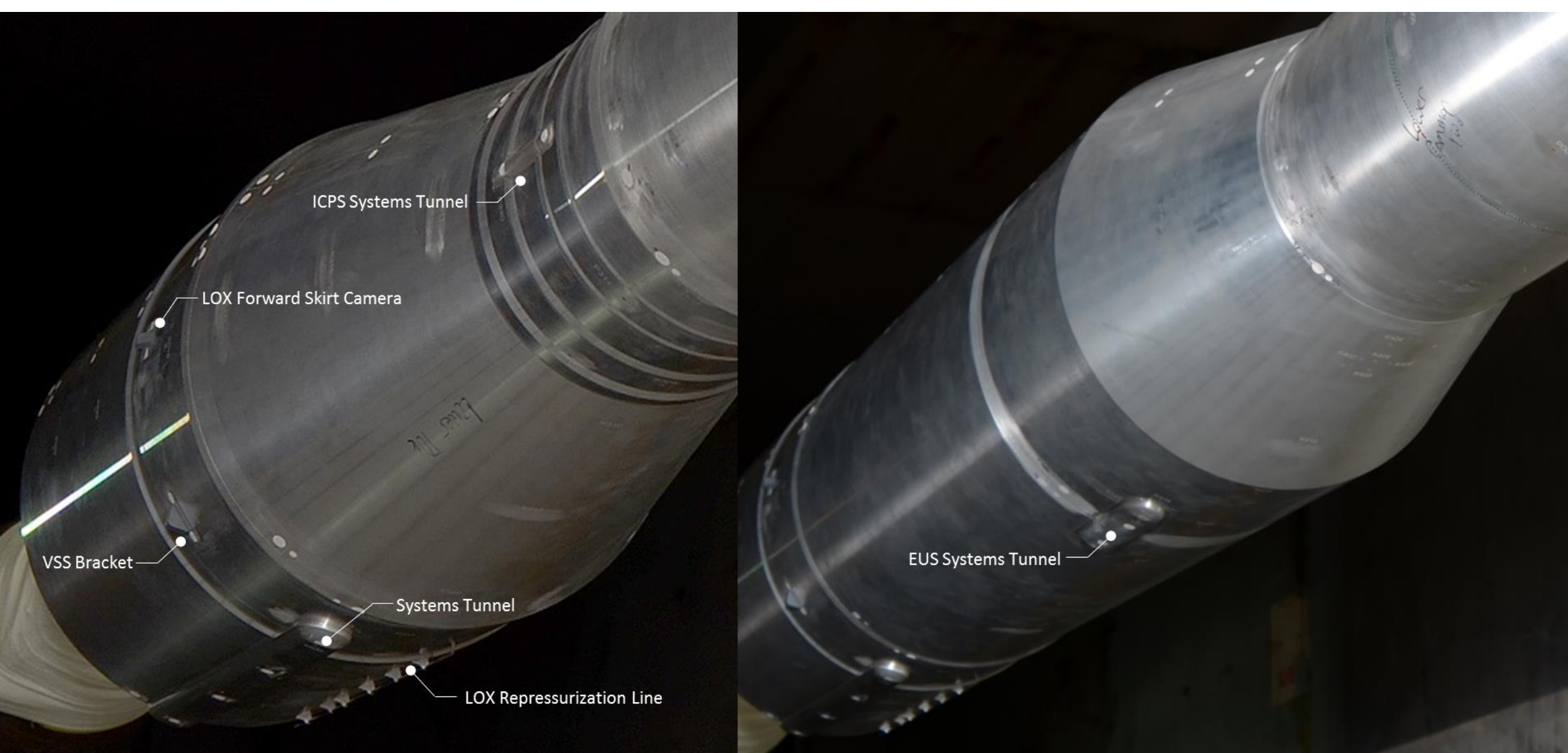


\section{Instrumentation}

- Dynamic Transducers

- Kulite Semiconductor Inc. XCL-072-5D and -15D ultra-miniature high frequency pressure transducer

- Included individual temperature compensation modules and Omnetics connectors

- Mounted via individually contoured holders

- Five cross-correlation patches

- Primarily newly purchased with additional needs

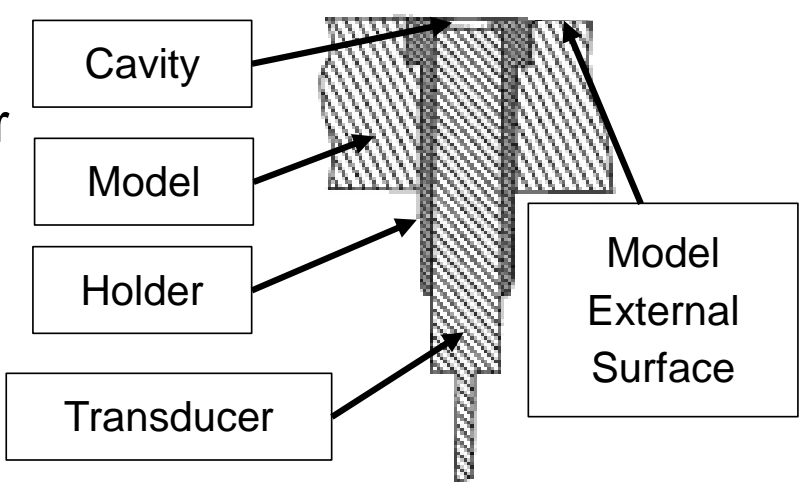
met using salvaged transducers

- ARC provided custom-built amplifiers located in sting

\section{$\checkmark$ Static Measurements}

- Mounted via individual contoured holders

- Two 64-channel electronically scanned pressure (ESP) transducer modules with temperature compensation

\begin{tabular}{|c|c|c|}
\hline Model & $\begin{array}{c}\text { Dynamic } \\
\text { Transducers }\end{array}$ & $\begin{array}{c}\text { Static } \\
\text { Measurements }\end{array}$ \\
\hline SLS-10003, 2.5\% & 161 & 39 \\
\hline SRB, 2.5\% & 60 & 23 \\
\hline SLS-27000, 2.5\% & 177 & 60 \\
\hline SLS-10003, 4\% & 65 & $62+9$ FADS \\
\hline SLS-28000, 4\% & 80 & $58+9$ FADS \\
\hline
\end{tabular}

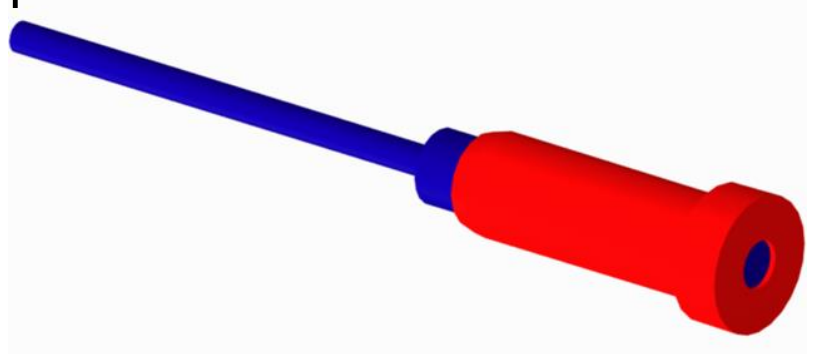




\section{Test Overview}

- Test Facility: NASA Ames Research Center (ARC) Unitary Plan Wind Tunnels (UPWT)

- 11' Transonic Wind Tunnel (TWT) : Mach $=0.70-1.40$

- 9'x7' Supersonic Wind Tunnel (SWT): Mach $=1.55-2.50$

- Facility Test Number: T11-0265, T97-0266, T11-0273, T97-0274

- Test Dates

- First Campaign: August $19^{\text {th }}-$ September $5^{\text {th }}, 2013$

- Second Campaign: December $16^{\text {th }}, 2013$ - January $8^{\text {th }}, 2014$

- Angle of Attack/Side Slip Angle: $\alpha / \beta=0^{\circ}, \pm 1^{\circ}, \pm 2^{\circ}, \pm 2.83^{\circ}, \pm 4^{\circ}, \pm 6^{\circ}$

- Mach Sweep: At $\alpha / \beta=0^{\circ}$ with full stack Block 1 model

- Reynolds Number:

- Primary: 3-million/foot

- Sensitivity study: 5-million/foot

- Sample Rates/Dwell Times Per Condition:

- $2.5 \%$ - High Frequency: $153.6 \mathrm{kHz} / 2.7$ seconds, Low Frequency $9.6 \mathrm{kHz} / 22$ seconds

- $4.0 \%$ - High Frequency: 102.4 kHz/4 seconds, Low Frequency 6.4 kHz/11.5 seconds

- High Speed Data Acquisition: National Instruments PXI based hardware

- 24-bit A/D cards

- $204 \mathrm{ksps}$

- DC coupled

- Manufacturer provided transducer calibration data used

$\checkmark$ Visualization

- Shadowgraph photographs and video

- Retro-reflective background oriented schlieren (RBOS) 


\section{Data Analysis}

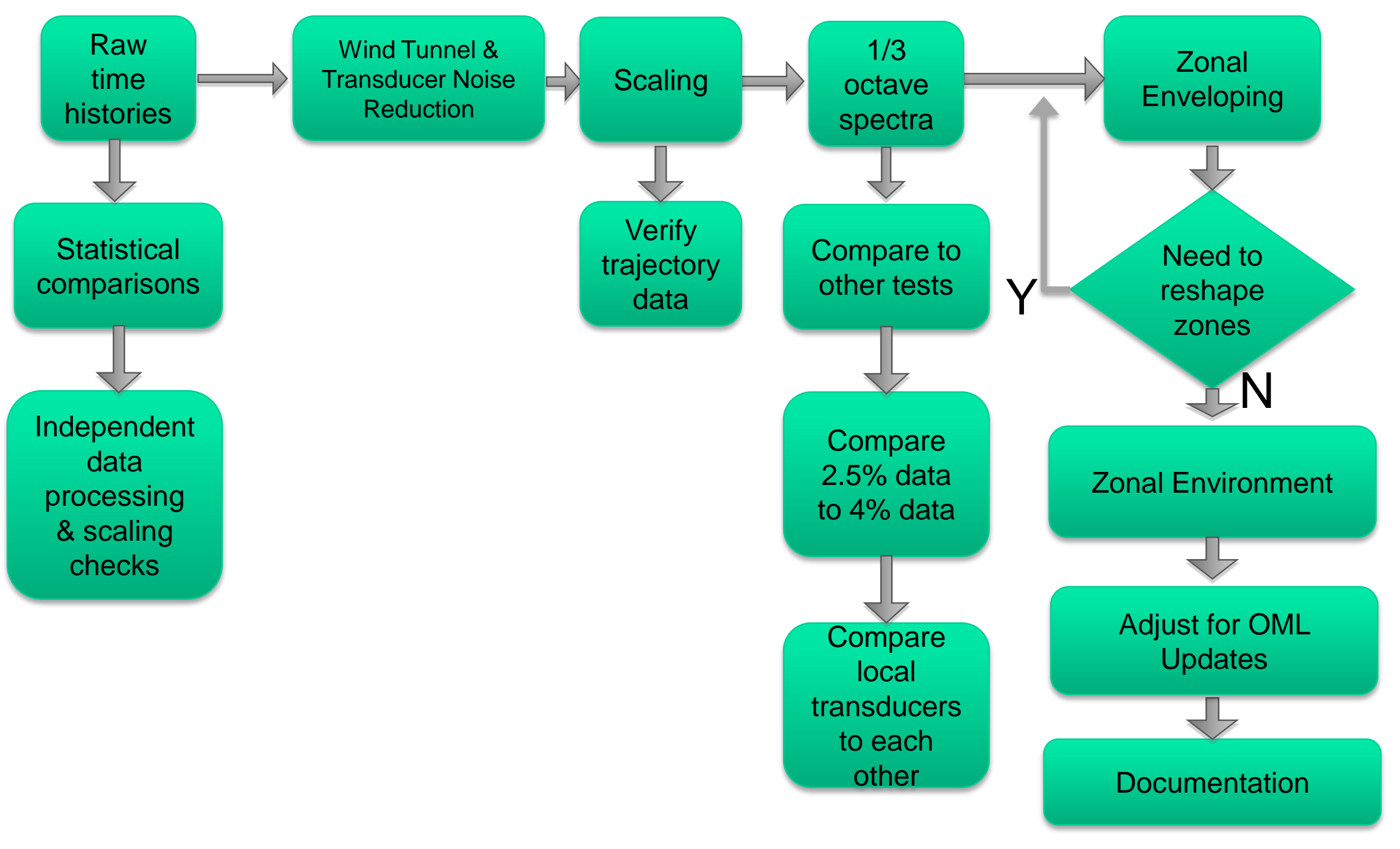




\section{Data Scaling}

- Amplitude

- Assume: $\left|\Delta C^{\prime}{ }_{p}\right|_{\text {flight }}=\left|\Delta C^{\prime}{ }_{p}\right|_{\text {tunnel }}$ where $\Delta C^{\prime}{ }_{p}=p_{r m s}^{\prime} / q_{\infty}$

- Therefore: $\mathrm{FPL}_{\text {flight }}=\mathrm{FPL}_{\text {tunnel }}+20 \log _{10}\left(\frac{q_{\infty} \text { flight }}{q_{\infty, \text { tunnel }}}\right)$

- Frequency

- Assume: $|S t|_{\text {flight }}=|S t|_{\text {tunnel }}$ where $S t=f l / U$

- Therefore: $f_{\text {flight }}=f_{\text {tunnel }}\left(\frac{l_{\text {tunnel }}}{l_{\text {flight }}}\right) \sqrt{\frac{T_{\text {flight }}}{T_{\text {tunnel }}}}$ 


\section{Results}

- Data shown for baseline configurations

- Standard SRB nose caps

- No AMO or BMO

- Full bandwidth zonal overall fluctuating pressure levels (OAFPL) as function of vehicle station (10-2000 $\mathrm{Hz}$ full scale)

- TWT Data: Mach $=0.7-1.4$

- SWT Data: Mach $=1.55-2.5$

- $\alpha \leq 4.47^{\circ}$

- Protuberance environments shown in the paper 


\section{General Acreage Environments}

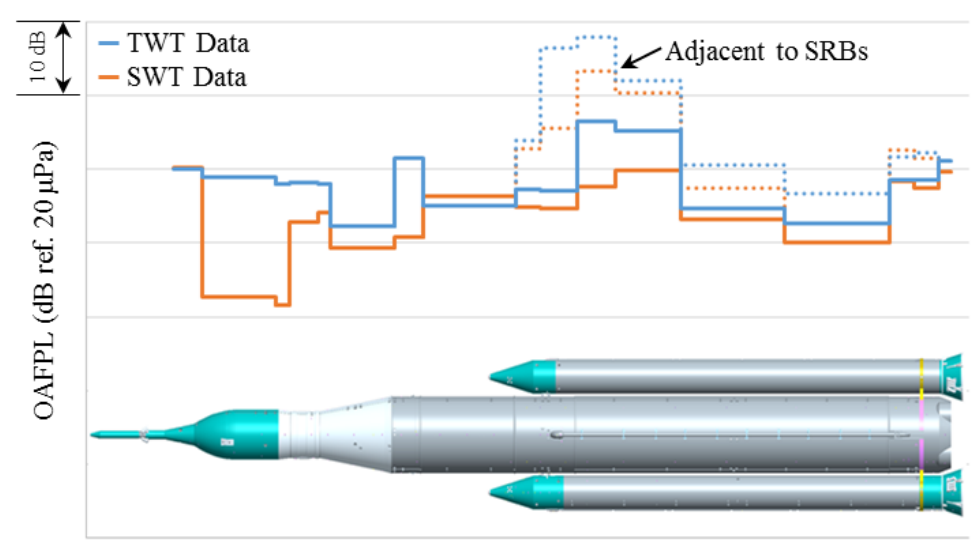

Vehicle Station

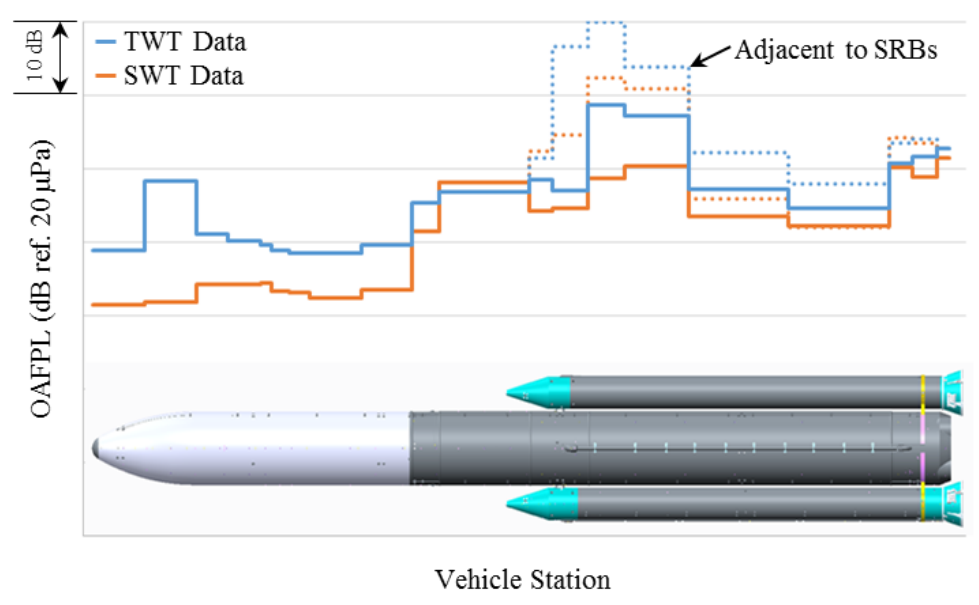

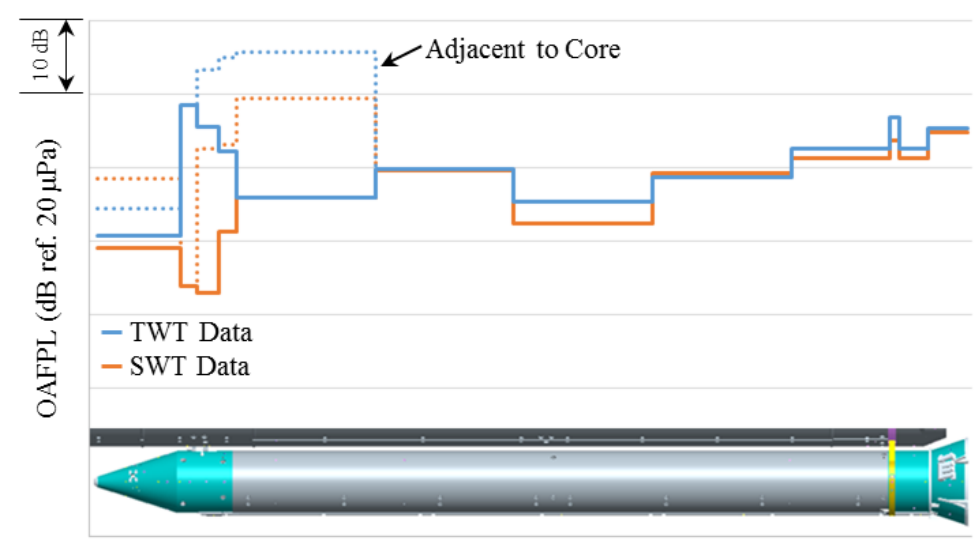

Vehicle Station

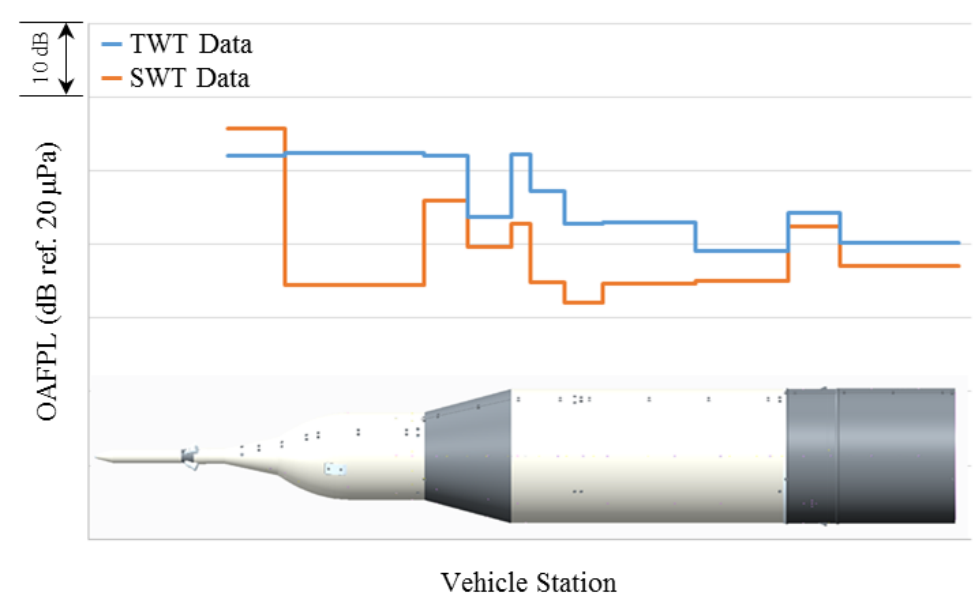




\section{Conclusions}

- All primary and secondary test objectives were accomplished with minimal transducer loss

- Primary Objective: Data have been used to successfully develop ascent aeroacoustic environments

- SLS-10005 Block 1 design environments released July 2014

- SLS-27004, SLS-27502, and SLS-28004 preliminary environments released August 2015

- Secondary Objectives: Data are being used for compartment venting environment determination, static CFD substantiation, unsteady CFD development

- Data are advancing the understanding of complex multi-body flow fields 


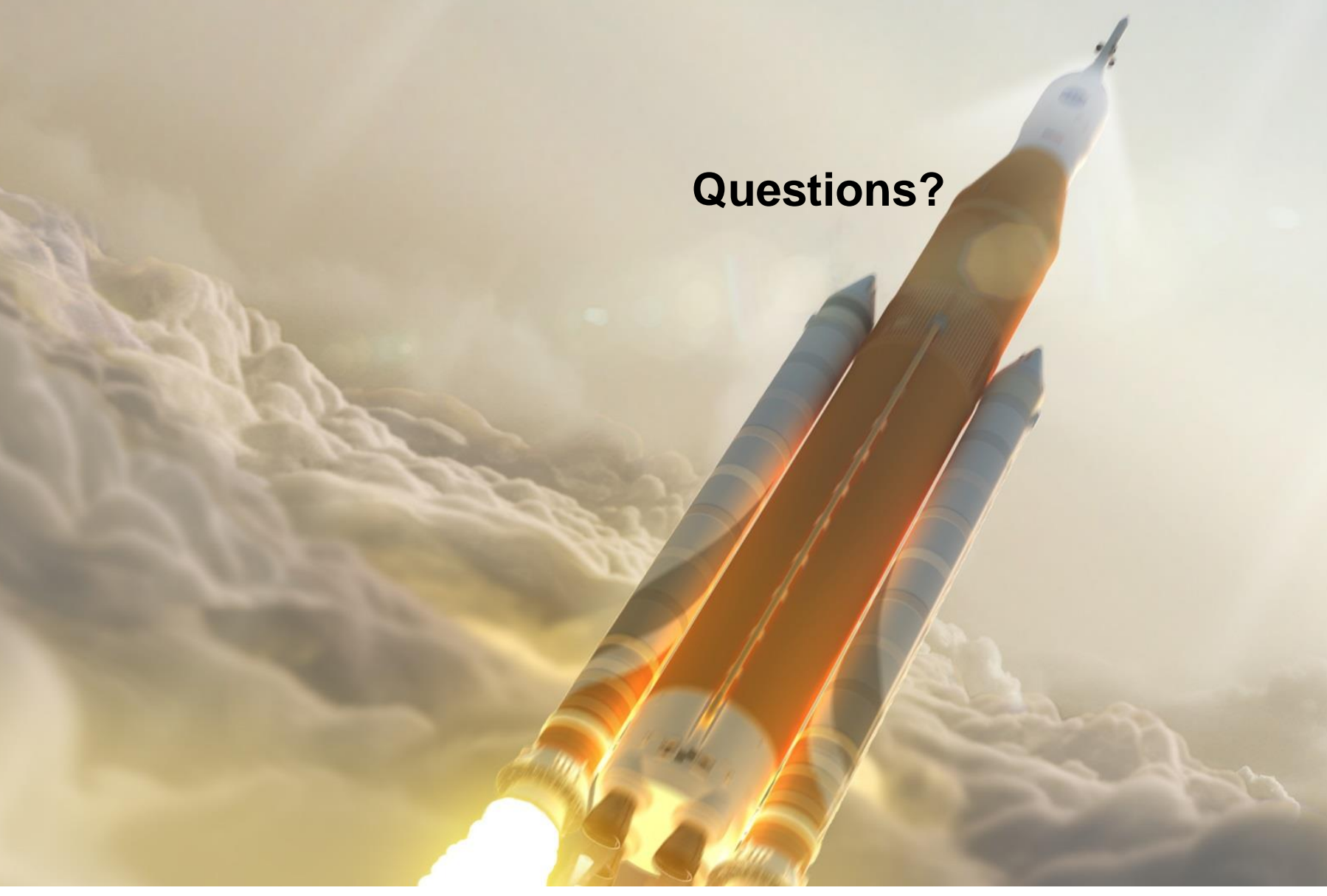

